\title{
AUTOEMPLEO Y EMPRENDIMIENTO. UNA HIPÓTESIS DE TRABAJO PARA EXPLICAR UNA DE LAS EXTRATEGIAS ADOPTADAS POR LOS GOBIERNOS PARA HACER FRENTE AL PROGRESO DEL MERCADO*
}

\author{
Germán Darío Valencia Agudelo* \\ Recibido: febrero 07 de 2012 • Aceptado: noviembre 13 de 2012
}

\section{RESUMEN}

Desde la década de 1990 se inicia una masificación de la cultura del emprendimiento en todo el mundo. Esta ha sido una estrategia implementada por los gobiernos, desde comienzos del siglo XX, para hacerles frente a problemas políticos, económicos y sociales que genera el progreso del mercado. Este artículo plantea la hipótesis de que los gobiernos fomentan la cultura del emprendimiento como un mecanismo para crear empleo y competitividad, a la vez que busca aumentar los ingresos de los ciudadanos para mejorar el bienestar social y la gobernabilidad. Para formular la hipótesis se hace una revisión de la literatura, donde se evidencia el interés creciente de los gobiernos, tanto locales como nacionales, por fomentar la cultura del emprendimiento a través de políticas públicas.

PALABRAS CLAVE

Emprendimiento, autoempleo, creación de empresas, políticas publicas

CLASIFICACIÓN JEL

L26, M13, 014

\section{CONTENIDO}

Introducción; 1. Smith y Cantillon: el progreso está en no tener que ocupar a todos; 2. La sociedad del 20:80 y el Tittytainment; 3 . autoempleo y mercado laboral; 4. La historia se repite; 5 . La consolidación del espíritu emprendedor: algunas explicaciones; 6 . Dinámica mundial de la relación entre gobierno y cultura del emprendimiento; 7 . Conclusiones y retos para la academia y el gobierno; Bibliografía.

\footnotetext{
El artículo de investigación es producto del proyecto de Investigación: "Estrategia para la sostenibilidad de los grupos A y Al para los años 2011 -2012", financiado por el Comité de Apoyo a la Investigación -CODI- de la Universidad de Antioquia y desarrollado por el grupo de investigación Hegemonía, guerras y conflicto del Instituto de Estudios Políticos de la misma universidad, con el cual se fomenta la investigación autónoma de los miembros del grupo. El autor agradece a los profesores Dany García Callejas y Jorge Iván González por sus valiosos aportes al texto, aunque los exime de cualquier responsabilidad sobre las ideas en él contenidas; lo mismo para los evaluadores anónimos de la revista.

** Economista, especialista en Gerencia Social y magíster en Ciencia Política, Universidad de Antioquia, Medellín, Colombia. Estudiante del Doctorado en Estudios Políticos de la Universidad Externado de Colombia. Profesor titular del Instituto de Estudios Políticos de la Universidad de Antioquia, Medellín, Colombia, y es miembro de los grupos de investigación Hegemonía, guerras y conflicto y Microeconomía aplicada de la misma universidad. Correo electrónico: german.valencia@udea.edu.co y gdvalencia@ yahoo.com.
} 


\section{SELF-EMPLOYMENT AND ENTREPRENEURSHIP. A LABOR HYPOTHESIS THAT HELPS EXPLAINING ONE OF THE STRATEGIES ADOPTED BY GOVERNMENTS FOR FACING MARKET PROGRESS \\ ABSTRACT}

Since the 1990's a massive culture around entrepreneurship has started worldwide. Governments have implemented this strategy since the beginning of the twentieth century, to face political, economic and social progress that the market generates. This article hypothesizes that governments promote the culture of entrepreneurship as a mean to create jobs and increase competitiveness, while seeking to increase the income of citizens and improve social welfare and governance. In order to formulate the hypothesis, a literature review is carried out, where the growing interest of both national and local Governments for sponsoring entrepreneurship can be evidenced through the public policies they apply.

\section{KEY WORDS}

Entrepreneurship, self-employment, business creation, public policies

\section{JEL CLASSIFICATION}

L26, M13, O14

\section{CONTENT}

Introduction; 1. Smith and Cantillon: Progress is in not needed to occupy everyone; 2. The 20:80 society and the Tittytainment; 3 . Self-employment and labor market; 4 . History repeats its self; 5 . The consolidation of the entrepreneur spirit: some explanations; 6 . World dynamic of the relation between government and entrepreneurial culture; 7. Conclusions and challenges for the academia and government; Bibliography.

\section{AUTOEMPREGO E EMPRENDIMENTO. UMA HIPÓTESE DE TRABALHO PARA EXPLICAR UMA DAS ESTRATEGIAS ADOTADAS PELOS GOVERNOS PARA FAZEREM FRENTE AO PROGRESSO DO MERCADO}

RESUMO

Desde a década de 1990 inicia-se uma massificação da cultura do empreendedorismo no mundo todo. Esta tem sido uma estratégia implementada pelos governos, desde os inicios do século XX, para fazer frente aos problemas políticos, económicos e sociais que gera o progresso do mercado. Este artigo planteia a hipótese de que os governos fomentam a cultura do empreendedorismo como um mecanismo para criar emprego e competitividade, à vez que procura aumentar os ingressos dos cidadãos para melhorar o bem estar social e a governabilidade. Para formular a hipótese se faz uma revisão da literatura, donde se evidencia o interesse crescente dos governos, tanto locais quanto nacionais, por fomentar a cultura do empreendedorismo a través de políticas públicas.

PALAVRAS CHAVE

Empreendedorismo, auto emprego, criação de empresas, políticas publicas

CLASIFICAÇÃO JEL

L26, M13, 014

\section{CONTEUDO}

Introdução 1. Smith e Cantillon: o progresso está em não ter que ocupar a todos; 2 . A sociedade do 20:80 e o Tittytainment; 3. Auto emprego e mercado laboral; 4. A historia se repeti: 5 . A consolidação do espírito empreendedor: algumas explicações; 6 . Dinâmica mundial da relação entre o governo e a cultura do empreendedorismo; 7. Conclusões e retos para a academia e o governo; Bibliografia. 
Autoempleo y emprendimiento. Una hipótesis de trabajo para explicar una de las extrategias adoptadas por los gobiernos ...

\section{INTRODUCCIÓN}

El fomento del emprenderismo, del empresarismo o del espíritu de creación de empresas son las distintas maneras de nombrar la cultura del emprendimiento empresarial, un fenómeno que viene imponiéndose en el contexto mundial desde la década de 1990, pero que proviene de la época de entreguerras en el siglo XX como estrategia diseñada por los gobiernos europeos y el norteamericano, para hacerle frente al desempleo que se extendía por aquel entonces. Hoy es común encontrar en casi todas las universidades de América Latina, por ejemplo, programas académicos que buscan fomentar la cultura del emprendimiento en los jóvenes estudiantes y en el conjunto de la sociedad o programas del Gobierno, ya sea local o nacional, que apoyan iniciativas de creación de empresas y el autoempleo.

Este fenómeno, que tiene una explicación simple, resulta aparentemente paradójico, pues nace en un contexto de fuerte intervención estatal y nuevamente reaparece en un momento donde prima la libre competencia. Tanto en aquel momento como en este, el fomento de la cultura del emprendimiento ha sido una de las estrategias de los gobiernos para hacer frente a problemas políticos y sociales que genera el progreso del mercado. A principios del siglo XX, los gobiernos promovieron el autoempleo como una forma política, económica y social de mantener ocupados en actividades provechosas para la sociedad a aquellos que no eran partícipes, por una u otra razón, de la maquinaria de guerra. Hoy, de nuevo, los gobiernos fomentan la cultura del emprendimiento como un mecanismo para crear empleo y competitividad, a la vez que busca aumentar los ingresos de los ciudadanos para mejorar el bienestar social y la gobernabilidad.

El presente artículo trabaja en esta última idea. Construye la hipótesis de trabajo de que los gobiernos apoyan la cultura del emprendimiento como una forma de hacer frente a los problemas sociales y políticos que el progreso del mercado les impone, problemas sociales como el desempleo y la falta de ingresos que inciden negativamente en la gobernabilidad y el mantenimiento del orden social. Los gobiernos locales y nacionales han encontrado en el emprendimiento un buen mecanismo de mantener ocupados a los ciudadanos, que ven, a su vez, esta propuesta como un mecanismo para salir de la pobreza y reducir su exclusión económica y social.

Para esbozar esta hipótesis se usa como instrumento metodológico la revisión de la literatura, la cual parte de algunos textos clásicos de economía y se detiene en la prolífera producción que hoy existe sobre el tema. Además, se hace un análisis crítico que permite, finalmente, dejar esbozada la hipótesis. De esta forma el artículo se divide en siete secciones: la primera plantea la idea de progreso de Adam Smith (1996 [1776]) y Richard Cantillon (1950 [1755]), la cual reducen a no tener que 
ocupar a todos en la economía. En la segunda, se presentan los avances de esta idea de progreso al finalizar el siglo XX, y los problemas sociales y políticos que ha generado, que según nuevo Braintrust Global, se solucionan con el Titytainment. La tercera presenta la dinámica mundial del mercado laboral y el autoempleo. La cuarta muestra, de manera breve, la historia del emprendimiento. La quinta enfatiza en las teorías que quieren explicar el surgimiento del emprendimiento en la época reciente. La sexta muestra la dinámica mundial en torno a la relación Gobierno y cultura del emprendimiento, y el rol que este actor cumple en el fomento de esta cultura. Finalmente, la séptima sección se concluye con el planteamiento de algunos retos que las políticas públicas deberán afrontar en el fomento y consolidación de la cultura del emprendimiento, pero antes se presentan algunas propuestas temáticas y metodológicas de cómo abordar el desarrollo de esta hipótesis.

\section{SMITH Y CANTILLON: EL PROGRESO ESTÁ EN NO TENER QUE OCUPAR A TODOS}

La mayoría de los economistas coinciden en afirmar que el libro La riqueza de las naciones de Adam Smith (1996 [1776]) dio inicio a la economía como ciencia. En este texto se exponen, de manera novedosa, tanto el origen como la naturaleza de la riqueza, y explica cómo la especialización del trabajo, la libertad económica y ampliación del mercado son los determinantes de la productividad y el bienestar social, ideas que fueron la base para la consolidación de la doctrina del liberalismo económico y del capitalismo. Pero tal vez una de las ideas más importantes desarrolladas por Smith (1996 [1776], p. 28) fue su pensamiento sobre el progreso. El ideal de sociedad "próspera y civilizada", para Smith (1996 [1776], p. 28) era aquella donde:

[... |numerosas personas no trabajan en absoluto y muchas consumen la producción de diez veces y frecuentemente cien veces más trabajo que la mayoría de los ocupados; y sin embargo, la producción del trabajo total de la sociedad es tan grande que todos están a menudo provistos con abundancia, y un trabajador, incluso de la clase más baja y pobre, si es frugal y laborioso, puede disfrutar de una cantidad de cosas necesarias y cómodas para la vida mucho mayor de la que pueda conseguir cualquier salvaje.

Lo contrario sucede en las sociedades "pobres y miserables", naciones salvajes que aunque ocupadas todo el tiempo en conseguir las cosas útiles para la vida de sí mismas y su familia o tribu, no conseguían alimentar a todos. Según Smith (1996 [1776], p. 28), en estos estadios del desarrollo las personas se ven "obligadas a veces a matar $y$ a veces a abandonar a sus niños, sus ancianos o a los que padecen enfermedades prolongadas para que perezcan de hambre o sean devorados por animales salvajes".

Las sociedades "civilizadas y prósperas" se caracterizan porque no todas las personas trabajan. Las que lo hacen son pocas y a pesar de ello el consumo de la 
Autoempleo y emprendimiento. Una hipótesis de trabajo para explicar una de las extrategias adoptadas por los gobiernos ...

sociedad es muy grande (10 veces y frecuentemente 100 veces mayor a las sociedades pobres y miserables). Su idea de progreso es marchar a una sociedad donde no todos trabajen y que con la producción de unos cuantos se pueda mantener toda la población y que incluso los pobres vean mejoradas sus condiciones de vida. En conclusión, según Smith (1996 [1776], p. 27), las sociedades civilizadas tenderán a mantener una población desocupada productivamente, debido a que con la división del trabajo y la especialización logra que una proporción pequeña de la población se dedique a producir "todo el suministro de cosas necesarias y convenientes para la vida que la nación consume anualmente".

Veinte años antes de aparecer esta idea esmithiana, Richard Cantillon (1950 [1755]), en su obra Ensayo sobre la naturaleza del comercio en general, ya se había atrevido a plantear algo muy similar, incluso a colocarle una proporción entre el número de personas que se requería que trabajaran para sostener a toda la población. En el capítulo 6 de la obra anteriormente referenciada, afirma que para mantener los estándares de vida en la Europa de la época, bastaba con que 25 personas adultas trabajaran para mantener a estas y 100 más. Ante esta afirmación, el mismo Cantillon (1950 [1755]) se encontró con un problema del progreso que Smith (1996 [1776]) no señalaría: el desempleo que provocaría esta situación, 100 personas quedarían desempleadas ¿Qué hacer con ellas?

Cantillon (1950 [1755]) se aventura a presentar una propuesta: se requiere del autoempleo y el empleo de diversión o de decoración. El autoempleo se genera por los empresarios o tomadores de riesgo: aquellas personas que por su afán de ganancia y por tener una idea que creen puede revolucionar el mundo son los invitados a autoemplearse. Con las demás personas se podrían alimentar los ejércitos de la patria, otros prestar servicios comunitarios y el resto tener empleos decorativos. Estos últimos se justifican en la medida que ayuden a la estabilidad social. Tras esta idea, Cantillon (1950 [1755]) reconoce que para mantener el orden y la gobernabilidad es necesario que el Leviatán o Estado promueva la ocupación en todos los pobladores que deseen.

\section{LA SOCIEDAD DEL 20:80 Y EL TITTYTAINMENT}

La historia, 250 años después, le ha dado la razón tanto a Adam Smith como a Richard Cantillon (1950 [1755]) en unos aspectos, aunque no en otros. La sociedad actual, efectivamente, se caracteriza por tener un número cada vez mayor de personas autoempleadas y con salarios bajos. El aumento de los niveles de productividad y tecnificación de las empresas ha provocado que el mercado laborar se ajuste y restructure. Los cambios en los sistemas productivos han generado un despido sistemático de trabajadores industriales, lo que ha obligado a estos a buscar opciones 
en el autoempleo, con lo cual se busca evitar una profundización de sus niveles de pobreza y desigualdad (Vázquez, 2005, p. 11)1.

Una explicación a esta dinámica del mercado laboral en las últimas décadas la ofrece John Gage, presidente de Sun Microsystems, en 1995. Cuando se le interrogó por la forma como toma las decisiones laborales en su compañía, este respondió que: "contratamos a nuestra gente por ordenador, trabajan en el ordenador y son despedidos también por ordenadores". Según el empresario, el éxito de su compañía está en que muy pocas personas son imprescindibles; esto a pesar de contar con más de 17.000 empleados. En su empresa -considera- son tal vez "seis, quizá ocho" los fundamentales, sin ellos "estaríamos perdidos". La conclusión de Gage es que casi 99\% de los empleados que tienen son prescindibles, sin ellos es posible el negocio (Martin y Schumann, 2000, p. 9).

En este diagnóstico sobre la situación laboral del mundo actual lo acompañó un grupo de 500 líderes mundiales, que en septiembre de 1995, se reunieron en el hotel Fairmont de San Francisco, Estados Unidos, para hablar del futuro de la economía; los llamados "el nuevo Braintrust Global" ${ }^{2}$. Ante la explicación de Gage, y después de varias intervenciones, los asistentes llegaron a una conclusión: "la visión de un ejército de parados inimaginables hasta hoy" (Martin y Schumann, 2000, p. 8). No es posible pensar un futuro caracterizado por suficientes puestos de trabajo, decentes y bien pagados en palabras de Martin y Schumann (2000, p. 8): "en el futuro, el 80 por ciento de la humanidad tendrá que vivir a expensas del 20 por ciento restante. No habrá puestos de trabajo para más". Es decir, según Gage, sin saberlo, se cumplen en la actualidad las predicciones esmithiana y cantillana.

Según Martin y Schumann (2000, p. 8), para los Braintrust Global el futuro se reduce "a un par de números y un concepto: '20 a 80' y Tittytainment". Proporción muy similar a la que intuyó Cantillon (1950 [1755]) y que Smith (1996 [1776]) no se atrevió a proponer. Es decir, de acuerdo con Martin y Schumann (2000, p. 10) "una quinta parte de todos los que buscan trabajo bastará para producir todas las mercancías y aportar las valiosas prestaciones de servicios que la sociedad mundial pueda permitirse. Ese 20\% participará, por tanto, activamente en la vida, el beneficio y el consumo, no importa en qué país".

Ante esta situación, el padre de la economía estaría muy a gusto, pues esto indicaría que la humanidad está situándose en el nivel de una sociedad próspera

\footnotetext{
${ }^{1}$ Situación ya advertida por Karl Marx (2001) en su análisis del sistema capitalista en El Capital, donde establece, bajo la figura de Ejército industrial de reserva, como debido a muchos factores en el mercado de trabajo, existe una masa trabajadora que está desempleada y disponible para su contratación.

2 Para tener una idea de quienes estaban, se encontraban reunidos George Bush, George Schultz y Margaret Thatcher, además de los presidentes de las principales compañías de comunicaciones (CNN y Ted Turnet, entre otras), y también importantes académicos de todo el mundo (Martin y Schumann, 2000, p.8).
} 
Autoempleo y emprendimiento. Una hipótesis de trabajo para explicar una de las extrategias adoptadas por los gobiernos ...

y civilizada, donde se requiere que unos cuantos trabajen y sostengan al resto. Sin embargo, como el mismo Cantillon (1950 [1755]) lo advirtió, el problema está en que no se sabe qué hacer con este $80 \%$ de población desplazada, desempleada y dispuesta a trabajar. En palabras de Rifkin (1995), el autor del libro El fin del trabajo, ese $80 \%$ de las personas estarán en sumos problemas.

Las posiciones frente esta situación fueron variadas en el seno del Braintrust Global. Según Martin y Schumann (2000, p. 10), para Scott McNealy, por ejemplo, citando Gage: "En el futuro, la cuestión será to have lunch to be lunch, comer o ser comido"; es decir, dejar que el mercado opere. Para otros, que también hacían parte del grupo reunido, la opción no debería ser esta; para Zbigniew Brzezinski, un geoestratega que fue consejero de Seguridad Nacional del presidente norteamericano Jimmy Carter, la solución es el Tittytainment (una palabra inglesa compuesta que combina: entertainment y tits -entretenimiento y pechos-); esta es una metáfora muy realista: la leche que brota del pecho de una madre lactante es la solución para entretener al bebe que llora. Según Brzezinski, citado por Martin y Schumann (2000, p. 11), "el buen humor de la frustrada población del mundo podría mantenerse con una mezcla de entretenimiento aturdidor y alimentación suficiente".

Así, la sociedad 20:80, en lugar de ser un gran logro como lo pensaba Smith (1996 [1776]), resulta convirtiéndose en un gran problema. Problema que según los empresarios presentes en la reunión: "deben ser otros los que se preocupen por los parados" (Martin y Schumann, 2000, p. 11). La opinión de estos grandes empresarios es que en los países desarrollados la tendencia es a que las personas limpien las calles por casi nada o encuentren un mísero refugio como trabajadores domésticos ${ }^{3}$. Al fin y al cabo, dice John Naisbitt, un futurólogo invitado a la reunión que relatan Martin y Schumann (2000, p. 11), "la era industrial, con su bienestar de masas, no es más que 'un pestañeo en la historia de la economía'." La humanidad tiene que habituarse a vivir en un mundo 20:80, donde los excluidos tendrán que ser calmados con el Tittytainment.

\section{AUTOEMPLEO Y MERCADO LABORAL}

Para ver el futuro del que hablaban en la reunión el nuevo Braintrust Global no tuvo que esperarse 250 años, como sí ocurrió con Adam Smith. Un año después de la reunión se podía constatar la tendencia en el mundo del trabajo. En solo Alemania, se hablaba de más de seis millones de personas que se encontraban dispuestas a

\footnotetext{
${ }^{3}$ Otras soluciones propuestas en el encuentro fue mantener ocupados a los desempleado como servidores voluntarios a la comunidad, la ayuda a los vecinos, la práctica de deportes o la participación en asociaciones de todo tipo; en palabras de Roy, citado por Martin y Schumann (2000, p. 11), "se podrían revalorizar estas actividades mediante una modesta remuneración y fomentar así la autoestima de millones de ciudadanos".
}

Semestre Económico, volumen 15, №. 32, pp. 103-128 • ISSN 0120-6346, julio-diciembre de 2012, Medellín, Colombia 
trabajar; igual ocurría en la mayoría de países de Europa. Para 1996, en Austria, las autoridades anunciaron que "cada año desaparecen 10.000 empleos industriales" (Martin y Schumann, 2000, p. 12). Desde mediados de la década de 1990 es cada vez más común encontrar una situación donde es difícil para la población encontrar empleos fijos, bien remunerados y donde las personas se encuentren satisfechas ${ }^{4}$.

¿Cuáles son las causas de esta tendencia en el empleo? La respuesta más recurrente es que debido al libre mercado y al desarrollo de tecnología se han reducido costos -entre ellos el de transporte- y esto hace que los salarios bajen. Un ejemplo típico de cómo opera la reducción de costos lo presenta el Consorcio Ford: desde mediados de la década de 1990, cada filial regional no desarrolla sus propios modelos, el producto terminado ya no se reelabora de una división a otra y se adapta en la tercera. Ha desarrollado una máquina empresarial global integrada que le permite desarrollar, comprar y distribuir online a escala mundial. Con ello se evita duplicar trabajo, incluso en las labores más simples en todas las sucursales. "El resultado son global cars con los que Ford establece una vez más el estándar mundial de cómo se puede llevar la fabricación de automóviles a la mayor eficacia posible" (Martin y Schumann, 2000, p. 124). La reestructuración le permite día a día ganar millonarias sumas, se reducen gastos exorbitantes y se suprimen varios miles de empleos de alta cualificación, bien pagados, de directivos, ingenieros y vendedores.

Al igual que en la compañía Ford, en el mundo laboral el trabajo cambia: "gremio a gremio, profesión a profesión, una revolución está transformando el mundo laboral. Casi nadie se libra" (Martin y Schumann, 2000, p. 124). Políticos y economistas buscan sin cesar sustitutos para este mar de trabajadores que dejan sus puestos. Urich Beck (1998) habla de que se está ante una sociedad del riesgo, una nueva sociedad caracterizada por que todos los puestos de trabajo están en peligro, incluso, los trabajos más tradicionales y seguros de la economía están en entredicho. Los oficinistas y administradores dudan que sus lugares de trabajo se encuentren en el futuro. Hoy es común hablar de trabajos ocasionales, provisionales, transitorios o en período de prueba. Se encuentran personas que sin querer van de empresa en empresa ocupando cargos provisionales, mediante contratos que no son renovados 5 .

Uno de los efectos más visibles de la globalización y liberalización económica está en el mercado laboral y, específicamente, en el cambio que sufre la estructura de la población ocupada. El trabajo autónomo o autoempleo viene convirtiéndose en

\footnotetext{
${ }^{4}$ Según la Organización Internacional del Trabajo -OIT- el desempleo en el mundo aumentó entre 2007 y 2009, entre 18 y 30 millones de trabajadores, incluso podría haber sido de 50 millones (OIT, 2009, p. 1).

${ }^{5}$ El mundo actual se caracteriza, desde la década de 1990, por las directrices del Consenso de Washington: desregulación del mercado, liberalización del comercio, libre movilidad de capitales, privatización de empresas y libre iniciativa privada en negocios como telecomunicaciones, transporte, bancos, etc.
} 
Autoempleo y emprendimiento. Una hipótesis de trabajo para explicar una de las extrategias adoptadas por los gobiernos ...

la principal característica del mercado laboral, a pesar de que aun contribuye poco a la creación de empleo y renta. Según Guzmán y Romero (2005, p. 80), el trabajo autónomo se difunde tanto en el mundo que comienza a ser tomado como la "forma primigenia de empresa y al trabajador autónomo como forma elemental de empresario". Desde la década de 1980 las tasas de autoempleo han crecido: un estudio realizado por Van Stel (2006) para 23 países de la Organización para la Cooperación y el Desarrollo Económicos -OCDE-, encontró un comportamiento de U en la tasa de autoempleo entre 1972 y 2002 (Gráfico 1).

Gráfico 1. Tasas de autoempleo en seis países de la OCDE0 (los propietarios de empresas por mano de obra)

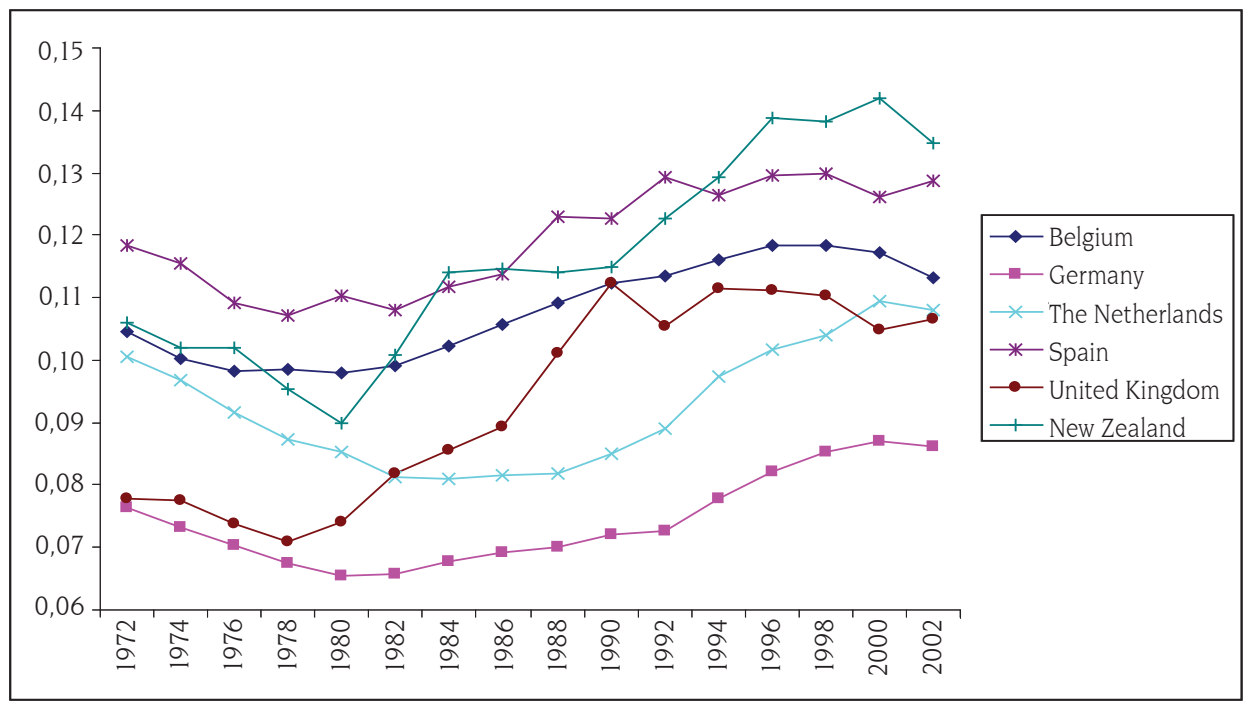

Fuente: tomado de Van Stel, André (2006).

Para 2003, la Organización Internacional del Trabajo -OIT- y la Organización para la Cooperación y el Desarrollo Económicos -OECD- calculaban las tasas de autoempleo así́: para los países desarrollados como Turquía, México y Corea del Sur esta tasas son superiores al 25\% (29,8\%, 29.7\% y 27,3\%, respectivamente); mientras que para los países en vía de desarrollo las tasas son superiores al 35\%

\footnotetext{
${ }^{6}$ Una aproximación a la definición sobre el concepto de autoempleo la realiza García (2002, p. 19): "la puesta en marcha de una actividad económica, por una o varias personas, con el objetivo fundamental de conseguir con ello una ocupación o puesto de trabajo, siendo precisamente el trabajo su principal aportación e interés en la empresa". Sin embargo, para la Organización Internacional del Trabajo, debido a que no dispone de datos que le permitan distinguir entre los trabajadores por cuenta propia y los empleadores autoempleo, engloba a estas dos categorías en la categoría de autoempleo.
} 
(algunos casos críticos son Vietnan, Colombia y Georgia donde las tasas son 41.1\%, $39,2 \%$ y $35 \%$, respectivamente), lo que significa, para el primer grupo, que uno de cada cuatro trabajadores se autoemplea, y, para el segundo, que este número casi se duplica (ver tabla 1).

Tabla 1. Tasa de autoempleo (autoempleados como porcentaje de la población ocupada) para varios países, 2003

\begin{tabular}{|c|c|c|c|c|c|}
\hline \multicolumn{6}{|c|}{ OCDE } \\
\hline País & $\%$ & País & $\%$ & País & $\%$ \\
\hline Alemania & 10,3 & Finlandia & 12,5 & Noruega & 7,0 \\
\hline Australia & 13,0 & Francia & 8,8 & Nueva Zelanda & 18,6 \\
\hline Austria & 10,7 & Grecia & $\ldots$ & México & 29,7 \\
\hline Bélgica & $\ldots$ & Holanda & $\ldots$ & Polonia & 21,8 \\
\hline Canadá & 9,5 & Hungría & 12,9 & Portugal & 25,2 \\
\hline Corea del Sur & 27,3 & Irlanda & 16,5 & Reino Unido & 12,0 \\
\hline Dinamarca & 8,1 & Islandia & $\ldots$ & Rep. Checa & 16,6 \\
\hline Eslovaquia & 9,6 & Italia & 23,4 & Suiza & 9,7 \\
\hline España & 17,0 & Japón & 10,5 & Suecia & 9,4 \\
\hline EEUU & 9,1 & Luxemburgo & 6,8 & Turquía & 29,8 \\
\hline \multicolumn{6}{|c|}{ Resto del mundo } \\
\hline País & $\%$ & País & $\%$ & País & $\%$ \\
\hline Argentina & 23,3 & Georgia & 35,8 & Rep. Macedonia & 8,2 \\
\hline Bulgaria & 9,6 & Hungría & 7,4 & Rumania & 21,2 \\
\hline Chile & 26,8 & Israel & 6,9 & Rusia & 5,7 \\
\hline Colombia & 39,2 & Kazajstán & 36,5 & Sri Lanka & 29,6 \\
\hline Costa Rica & 19,3 & Letonia & 6,1 & Singapur & 8,1 \\
\hline Croacia & 15,6 & Malasia & 15,6 & Taiwán & 27,6 \\
\hline Ecuador & 29,1 & Marruecos & 26,1 & Tailandia & 31,5 \\
\hline El Salvador & 28,0 & Panamá & 28,6 & Uruguay & 25,0 \\
\hline Estonia & 5,7 & Perú & 33,9 & Vietnam & 41,1 \\
\hline
\end{tabular}

Fuente: OCDE y OIT tomado de Guzmán y Romero (2005, p. 84).

Finalmente, hay que destacar que tal crecimiento del autoempleo se da básicamente en las actividades industrial y de servicios: entre 1983 y 2003 se nota un incremento en las tasas de autoempleo en la mayoría de países de la OCDE (en Alemania pasó de 7,06\% a 9,48\%; en Corea del Sur de 19,06\% a 19,76\%, en Irlanda de 
Autoempleo y emprendimiento. Una hipótesis de trabajo para explicar una de las extrategias adoptadas por los gobiernos ...

$8,9 \%$ a $11,8 \%$ y en Italia de $18,1 \%$ a $21,18 \%)$. En síntesis, el autoempleo en el mundo es un fenómeno generalizado y en crecimiento.

\section{LA HISTORIA SE REPITE}

Desde mediados del siglo XIX, en plena época de librecambio, comenzó el mundo empresarial a caracterizarse por el nacimiento de los Trus o monopolios. Las empresas grandes se consumían a las pequeñas, la producción a gran escala y la elasticidad precio provocaban que las pequeñas empresas sucumbieran y desaparecieran a causa de las ineficiencias económicas. Economistas como Chandler (1977), Galbraith (1956) y Schumpeter (1942) advertían que las pequeñas empresas tenían la pelea perdida con las grandes, debido a sus propias ineficiencias.

Debido a esta situación, se creía que el fin de las pequeñas empresas había llegado. Pero eso no fue así. Los gobiernos colocaron por encima de la eficiencia económica las razones sociales y políticas. Según Audretsch y Thurik (2000), se comenzó a priorizar la creación de empresas, aunque fueran pequeñas, ya que era esencial para el mantenimiento del sistema político. El gobierno, preocupado con la situación de las personas que no se relacionaban con la economía de guerra, vio en la promoción del espíritu empresarial una buena oportunidad de crear empleo. Las pequeñas empresas eran el principal proveedor de empleo y, por ende, de la estabilidad social y política.

Los gobiernos de Europa, pero también el norteamericano, promulgaron entre sus ciudadanos el autoempleo. Una forma política, económica y social de mantener ocupados en actividades provechosas para la sociedad aquellos que no eran partícipes de la maquinaria de guerra (por una u otra razón). Pequeñas empresas familiares, conformadas, principalmente, por mujeres, ancianos, lisiados y niños surtían de algunos productos básicos y fundamentales a los canales de distribución de las fuerzas armadas, al Gobierno y a otras instituciones que no estaban al servicio de la guerra. Esta iniciativa mostró ser eficiente en concentrar a estas microempresas en otras preocupaciones distintas a la guerra.

En Estados Unidos, por ejemplo, el presidente Jefferson apoyó la formación de un espíritu empresarial en la ciudadanía. Fue clave para ello la aprobación de la Ley Robinson-Patman, que proporcionó una cierta medida de protección a los pequeños minoristas independientes y sus proveedores independientes de la competencia desleal y de la integración vertical que tenían luego del período del librecambio. Con esto, se provocó en Estados Unidos la creación de un gran número de pequeños negocios, convirtiéndose en la mejor respuesta política para proteger las pequeñas empresas, menos eficientes y poco viables. 
A partir de este momento, y con la transformación que sufre el Estado después de la época de la Gran Depresión, este asume la estrategia de comandar la economía, fomentarla, mantener la estabilidad y darle orden social. El fomento de la cultura del emprendimiento era un buen mecanismo para lograrlo. Esta dinámica de apoyar las iniciativas privadas para la creación de empresas se mantuvo durante todo el siglo XX; el Gobierno norteamericano conservó el interés, aunque cada vez menor en el surgimiento de negocios, en el autoempleo como estrategia para mantener a todos ocupados, tarea que complementó con la suya de dar trabajo en las empresas del Estado.

Con la crisis del Estado, la reestructuración de la economía y el fomento del mercado, en la década de 1980, se pensó que de nuevo las grandes empresas se tomarían el mundo, que este sí sería el fin de las pequeñas empresas, que la globalización pondría fin a un convaleciente que el Gobierno había mantenido con vida por la necesidad política de ayudar a mantener la gobernabilidad. Sin embargo, la cultura del emprendimiento empresarial se ha popularizado aún más ${ }^{7}$. Se asiste de nuevo a un resurgimiento de la cultura del emprendimiento en el mundo. Los pequeños negocios pululan por todas partes, miles de iniciativas son apoyadas diariamente por el Gobierno, por las universidades o por organismos internacionales. Así, como el Ave Fénix, de nuevo las pequeñas empresas resisten el embate neoliberal y renacen con fuerza.

\section{LA CONSOLIDACIÓN DEL ESPÍRITU EMPRENDEDOR: ALGUNAS EXPLICACIONES}

La consolidación del espíritu empresarial ha hecho también que resurjan los estudios que buscan explicar las causas de este fenómeno. Como se advirtió en la primera sección, el espíritu empresarial ha sido de interés de la economía desde los primeros economistas, y hoy hay una gran parte de ellos ocupados del tema del emprendimiento (Godin, Clemens y Veldhuis, 2008; Rodríguez y Jiménez, 2005). La historia se puede sintetizar en tres visiones: la del empresario innovador de Schumpeter (1934; 1942), la de la búsqueda de ganancia y oportunidades de Kirzner (1997) y la de agentes amantes del riesgo de Knight (1999). La más conocida es la Schumpeter $(1934 ; 1942)$, quien plantea el surgimiento del espíritu empresarial o "empresario innovador" como una forma de los empresarios crear nuevos productos y procesos para hacer frente a la dinámica del mercado (Wennekers y Thurik, 1999). Le sigue el austriaco Kirzner (1997), para quien la iniciativa empresarial es una buena oportunidad de conseguir grandes beneficios ante un mercado con muchas oportu-

\footnotetext{
${ }^{7}$ Los análisis internacionales mostraban que las pequeñas empresas eran una realidad en todas partes, aunque con mayor prevalecencia en Europa y América del Norte, e, incluso, aumentado en las últimas décadas (Acs y Armington, 1993; Blau, 1987; Loveman y Sengenberger, 1991).
} 
Autoempleo y emprendimiento. Una hipótesis de trabajo para explicar una de las extrategias adoptadas por los gobiernos ...

nidades. Y finaliza la propuesta del norteamericano e institucionalista Knight (1999), que muestra al espíritu empresarial como un comportamiento de las personas que les gusta asumir riesgo en contextos inciertos (Shane, 2000).

Otras explicaciones más recientemente son las de Carlsson (1989; 1992), quien presenta dos explicaciones al resurgimiento del espíritu empresarial: una, la amplia competencia económica y la fragmentación del mercado ocurrido después de la década de 1970; y dos, los avances tecnológicos. Piore y Sable (1984) sostienen que la inestabilidad del mercado de la década de 1970 dio lugar a la desaparición de la producción en masa y la promoción de la especialización flexible: el desarrollo tecnológico generó importantes deseconomías de escala y reorientó las actividades económicas a otras no manufactureras. Brock y Evans (1989) destacan el mayor nivel de educación de la población, cambios en los hábitos de consumo y transformaciones en el mercado de trabajo como mayor flexibilización y movilidad laboral. Y Audretsch y Thurik (2000) enfatizan en la creciente mundialización, que ha cambiado la ventaja comparativa de los países económicamente avanzados hacia el conocimiento basado en la actividad económica; pero también, hablan que los altos niveles de desempleo estimulan la creación de empresas (efecto refugiado o refugee efect) (Audretsch, Carree y Thurik, 2001).

Finalmente, están los trabajos que destacan el papel clave que han cumplido los gobiernos en la promoción de la cultura del emprendimiento. Loveman y Sengenberger (1991) subrayan el papel de las políticas públicas para promover el sector de pequeñas empresas, políticas que buscan disminuir los costos de transacción. ACS y Armington (2004) y Foelster (2000) ven en el fomento del espíritu empresarial una vía del Gobierno para generar innovación, dinamizar la industria y generar empleo. Y Audretsch y Thurik (2000) muestran cómo el espíritu empresarial es fruto de un conjunto de fuerzas y factores, donde las legales e institucionales, así como los factores culturales y sociales, son claves para el fomento.

Igualmente, se encuentran los trabajos de Saxenian (1990; 1994) que sostienen que los entornos empresariales son fruto de la red de ayudas que Gobierno y la sociedad les dan a estas en términos de instituciones que apoyen en el aprendizaje y lo financiero; se crea confianza que permite fortalecer las relaciones con el entorno, el contexto le ofrece recurso y relaciones para el desarrollo (Johannisson, 1995). Baumol (1990) muestra cómo el marco institucional es crucial para crear impactos positivos en el espíritu empresarial y el progreso económico. Y los de Grossman y Helpman (1991), Lucas (1988; 1993) y Romer (1986) ven el resurgimiento del espíritu empresarial como un importante motor del crecimiento, donde el Gobierno a través de sus políticas ha logrado impulsar la difusión del conocimiento y el apoyo a la generación de innovaciones. 
En América Latina y el Caribe los estudios sobre el fenómeno de la iniciativa emprendedora los han tenido una rápida expansión. Se destacan los trabajos de Álvarez y otros (2010), Amorós y Cristi (2008), Amorós, Fernández y Tapia (2012), Kantis (2004; 2005a) y Kantis, Ishida y Komori (2002) y Tiffin (2004). Por ejemplo, Capelleras y otros (2010), Peres y Stumpo (2002) y Tiffin (2004) han mostrado las múltiples repercusiones que ha tenido el tema del emprendimiento en la región, en particular, en el ámbito de las políticas públicas, que buscan crear nuevas empresas, aumentar el autoempleo y mejorar el desarrollo social y económico.

En síntesis, existe en la literatura una gran variedad de trabajos que enfatizan en uno o varios factores que contribuyen al impulso del emprendimiento. Por ejemplo, en el proceso de globalización, en las reformas a los mercados de la década de 1990, en las políticas intervencionistas del Estado en la economía, en los procesos de flexibilización laboral, en la tercerizaciones de la producción, en la innovación tecnológica y en la transformación de los asalariados en empresarios; incluso, como se ha visto, resaltando las precarias condiciones socioeconómicas de pobreza y la sobrevivencia de la población latinoamericana como explicación de su aparición y desarrollo.

\section{DINÁMICA MUNDIAL DE LA RELACIÓN ENTRE GOBIERNO Y CULTURA DEL EMPRENDIMIENTO}

En los últimos 30 años (1980-2010) los gobiernos de todo el mundo se caracterizan por haber realizado cambios radicales a su política económica: se han reducido o eliminado algunas de las políticas industriales de fomento, de producción de bienes y servicios y de intervención en los diferentes sectores productivos. De acuerdo con Vázquez (2005, p. 14), los gobiernos se han centrado en mantener estable el entorno macroeconómico, con políticas fiscales y de inversión no intervencionista, que contengan el déficit público y dejen a los privados tomar sus decisiones. Según Valencia (2004), se ha pasado de un Estado empresario a uno regulador.

Adicionalmente, el mundo se caracteriza por un fenómeno expansivo de la globalización y una apuesta por desarrollo del mercado como estrategia para crecer. Los ciudadanos quieren que la globalización sea "un éxito en beneficio de todos" (Martin y Schumann, 2000, p. 17). Por ello es común encontrar, en cualquier región, protestas ciudadanas contra los gobiernos y políticos para que se les tenga en cuenta en esta dinámica económica, por ejemplo, mediante políticas de generación de empleo. Los gobiernos son conscientes de que tener una cantidad cada vez mayor de personas sin trabajo y sin seguridad social es problemático; esto tiene un costo político demasiado alto. Ese $80 \%$ de la población que está excluido, está descon- 
Autoempleo y emprendimiento. Una hipótesis de trabajo para explicar una de las extrategias adoptadas por los gobiernos ...

tento en sus casas y requiere trabajar. Sus votos pesan mucho. Las democracias se comportan como mercados, se compra y se vende, y el medio de pago es el voto. Los ciudadanos saben que tienen poder de votación y lo utilizan a favor o en contra de los gobernantes.

En este contexto y ante las insuficiencias de las políticas macroeconómicas para resolver los problemas asociados a la creación de empleo, aumento en la producción, aumento de mercados y mejorar el bienestar social, los actores locales, de forma espontánea, han tratado de encauzar los procesos de ajuste, mediante acciones que se proponen atender a un problema global. Según Vázquez (2005, p. 41), los gobiernos locales han tenido que asumir las responsabilidades del Gobierno nacional en términos de apoyo a sectores estratégicos y creación de empresas: "se plantearon la necesidad de mejorar la respuesta de los sistemas productivos locales a los desafíos que significan el aumento de la competencia en los mercados y los cambios de la demanda". Sobre todo, han tenido que asumir el reto de mantener la gobernabilidad desde lo local, dando respuesta a las demandas sociales, políticas y económicas.

Muchos de los gobiernos locales utilizan como estrategia para dar respuesta a estas demandas, la gobernanza o gobernación del desarrollo, que consiste en un proceso de cooperación y coordinación que integre las estrategias de los actores públicos y privados, sus decisiones de invertir y los intercambios que se establecen entre ellos. Uno de los programas más utilizados y más funcionales, por parte de los gobiernos locales para dinamizar la producción, mantener o mejorar el nivel de empleo y remediar la exclusión económica ha sido el fomento de la cultura del emprendimiento. Para Vázquez (2005), el fomento del emprendimiento o la creación de empresas ha sido uno de los estímulos que aplican las organizaciones públicas en el territorio para estimular los procesos de desarrollo endógeno.

Los gobiernos locales han introducido innovaciones al tejido productivo, y han creado las condiciones institucionales y espaciales que favorecen el desarrollo de iniciativas productivas. Es común encontrar en todo el mundo, gobiernos locales que a través de sus agencias apoyan financieramente la creación de empresas, prestan servicios de asesoría, premian iniciativas y estimulan la formación del espíritu emprendedor ${ }^{8}$. En América del Norte, por ejemplo, se creó a partir de 1990, una serie de instituciones con la intención de desarrollar el espíritu empresarial. Lo

\footnotetext{
${ }^{8}$ Un caso representativo de colaboración entre gobierno y creación de empresas es China. Sobresale el Programa de Incubación -Torch-, creado en 2000 y administrado por el Ministerio de Ciencia y Tecnología. En sus primeros años este programa maneja 110 incubadoras, con más de 5.000 empresas, graduando a 1.785 empresas y creando 170.000 empleos, y para 2006 se calculaban 200 incubadoras. China ha convertido las incubadoras en un tema estratégico dentro de sus planes quinquenales, e importantes fondos del gobierno han sido asignados para el siguiente lustro.
} 
mismo ha ocurrido en América Latina donde, desde finales de la década de 1990, se ha presentado un interés creciente del Gobierno por la creación de empresas; para Figarella y Zamora (2006, p. 55), "la cultura emprendedora busca cambiar los cánones establecidos y generar, a partir de innovación, métodos más efectivos de ejecutar los negocios".

Para lograr la gobernanza y la creación de la cultura del emprendimiento, los gobiernos locales se han apoyado en las universidades, en agencias internacionales de desarrollo y en el mismo sector productivo. En las universidades han encontrado un buen aliado en la formación del espíritu emprendedor; a través de ellas se les ofrecen a los emprendedores: asesorías, consultas profesionales, simuladores computarizados e instrumentos metodológicos que orientan a estas personas a la concreción de una idea de negocio. En América Latina, por ejemplo, es pionero el Tecnológico de Monterrey, que lidera el Modelo de Transferencia del Programa Emprendedor, donde el 68\% de sus egresados se han formado como emprendedores 9 .

En cuanto a las agencias internacionales de desarrollo se tiene el apoyo del Banco Interamericano de Desarrollo -BID-, el Sistema Económico Latinoamericano y del Caribe -SELA- y la Organización de las Naciones Unidas para la Educación, la Ciencia y la Cultura -Unesco-, quienes apoyan el espíritu empresarial. Un ejemplo es el Programa Bolívar, creado en Caracas en 1992, por estos organismos, y con el apoyo del Gobierno venezolano y de otras organizaciones, y cuya estrategia principal es el fomento de la creación de empresas. Para ello crearon, en 1998, el Instituto Internacional de Fomento Empresarial -Infoem- que busca fortalecer el tejido empresarial de la pequeña y mediana empresa, a través de políticas de fomento que impulsen el desarrollo de nuevos emprendimientos.

Otros programas son: el Programa de Estímulo de la Capacidad Emprende dora -Pece-, creado en América Latina, en 1999, como una respuesta práctica a la necesidad de formar, orientar y apoyar el surgimiento de "empresarios y empresas de futuro", cuya estrategia es la de sensibilizar, promover y desarrollar el espíritu emprendedor. Y el Programa Andino de Competitividad -PAC- cuya intención es, a través de los emprendedores, introducir innovaciones que aceleren la producción de

\footnotetext{
${ }^{9}$ El 30\% de las universidades de Latinoamérica trabajan el tema emprendedor en la estructuras curriculares, demás el 70\% de ellas trabajan el tema en sus programas de extensión y bienestar estudiantil, según cifras del Congreso Internacional de Emprendedores 2006 realizado en Guadalajara, México, por el Instituto Tecnológico de Estudios Superiores de Monterrey. En Colombia, el número de universidades que apoyan estos programa es grande: La Universidad de Antioquia con su programa Gestión Tecnología (que apoya el emprendimiento empresarial y transferencia tecnológica), la EAN (con su programa Emprendedor ha hecho que el 25\% de sus egresados sea empresarios), el Icesi de Cali (que tiene un Centro de Desarrollo del Espíritu Empresarial (Varela y Bedoya, 2006)) y la Cátedra Virtual de Creación de Empresas de Base Tecnológica, que se trabaja vía Internet a estudiantes universitarios de todo Colombia (Figarella y Zamora, 2006, pp. 56-57).
} 
Autoempleo y emprendimiento. Una hipótesis de trabajo para explicar una de las extrategias adoptadas por los gobiernos ...

bienes y servicios, pues se reconoce que en los países andinos hay una alta tasa de actividad emprendedora, pero que esta no se ha traducido en importantes tasas de crecimiento de las economías, aunque dicha actividad es más bien de subsistencia ante la ausencia de oportunidades de empleo dependiente ${ }^{10}$.

Finalmente, y después de varios años, se le ha sumado a estas iniciativas locales el apoyo del Gobierno nacional. El Gobierno central ha diversificado su estrategia de ayuda a la generación de la cultura del emprendimiento a través de otorgamiento de créditos, ayudas técnicas, creación de incubadoras de empresas y simplificación de los trámites para la creación de nuevas empresas. Vienen basándose en la idea de que "quienes crean riqueza son los empresarios, los estados facilitan y crean el ambiente propicio para que los emprendedores busquen formar la vía de la creación de empresas como proyecto de vida" (Kantis, 2005, citado por Crissién, 2006, p, 105).

En Colombia, por ejemplo, el Gobierno Nacional a través de la Ley ha orientado al fomento de la cultura empresarial; así, la Ley 1014 de 2006 (CRC, 2006) obliga a la Educación Básica a impartir cursos de emprendimiento. También ha creado programas como el Fondo emprender, Fomipyme, los Comités Asesores Regionales para el Comercio Exterior (Carces) y el programa Jóvenes Emprendedores Exportadores (Emprendedores Colombia) ${ }^{11}$. En Bolivia, el Gobierno, a través del Ministerio de Asuntos Campesinos, Indígenas y Agricultura, lleva a cabo el Programa LiLL indí gena, destinado a apoyar los emprendimientos de las Organizaciones Económicas Campesinas (Antelo, 2006, p. 44); y también el Ministerio de Educación, mediante la Unidad de Coordinación de Políticas y Fortalecimiento de la Educación Técnica y Tecnológica, impulsa la capacidad emprendedora de los estudiantes.

Una de las acciones más utilizadas por los gobiernos para desarrollar la cultura del emprendimiento ha sido mediante las incubadoras de empresas ${ }^{12}$. Esto debido

\footnotetext{
${ }^{10}$ El Banco Mundial ha otorgado aproximadamente US\$ 10.000 millones en las últimas tres décadas. El Banco Interamericano de Desarrollo - BID- ha apoyado el fortalecimiento institucional de la Fundación Endeavor en Uruguay, a la capacidad emprendedora de la Fundación Ciudad del Saber en Panamá. También la OECD apoyo a la capacidad emprendedora y crecimiento de las pequeñas empresas y el desarrollo local (promoción de la capacidad emprendedora, generación de empleo y desarrollo económico local) y la OIT, la Unesco y la FAO con el apoyo a Pymes.

${ }^{11}$ Estas iniciativas estatales se han visto apoyadas por los medios de comunicación como la revista Dinero que organiza un concurso anual para otorgar premios a quienes presenten mejores y más viables ideas de negocios, optimizando el clima del emprendimiento. A 2006 se hacían cálculos de 800 proyectos de nuevas empresas que sobrevivían después de las tres convocatorias que el Fondo Emprender había realizado (Crissien, 2006, p. 108).

${ }^{12}$ En Estados Unidos, por ejemplo, se calculaba más de 900 incubadoras de empresas, que han creado 19.000 empresas y 245.000 puestos de trabajo. En el mundo se pasó de 18 incubadoras de empresas en 1980 a 900 en 2002 y hoy se calcula cerca de 4.000. En Europa se calculan mil incubadoras que generan 40.000 puestos de trabajo cada año. En cuanto a los países en desarrollo se hablaba de cerca de 500 incubadoras en 1997, pero a partir de ese año la tasa de crecimiento anual de estas se calcula en un
} 
a que esta estrategia permite que las nuevas empresas sean más viables y sostenibles -se calcula que la supervivencia de las empresas incubadas es de $80 \%$ a $85 \%$, mientras las no incubadas alcanzan una tasa del 30\%-. Este proceso dura entre tres y cinco años para cada empresa (Delvalle, 2006, p. 15). En Colombia, el caso más representativo de incubación de empresas es el Sistema Nacional de Incubación, a cargo del Servicio Nacional de Aprendizaje -Sena-. En general, el Gobierno sabe que las incubadoras de empresas logran promover el desarrollo regional y descentralizado, articular cadenas productivas y dinamizar los clusters regionales, generar empleo, redistribución de la renta y oportunidades económicas.

El resultado, después de más de una década de apoyo, tanto de los gobiernos locales y nacionales, como de organismos internacionales, de las universidades y del sector productivo ha sido la gestación de una cultura del empresarismo en todo el mundo. ${ }^{13}$ En América Latina y el Caribe este impacto es creciente y significativo; la cifras muestra un crecimiento cada vez mayor de la actividad emprendedora (tabla 2). En unos países más que en otros como Ecuador y Argentina, en este último, avivado por la crisis económica y financiera que soporta en la última década, donde el autoempleo y el emprendimiento se han convertido en un movimiento anticíclico.

Tabla 2. Evolución de la actividad emprendedora en América Latina y el Caribe, 2000-2010

\begin{tabular}{|l|c|c|c|c|c|c|c|c|c|c|c|}
\hline País & 2000 & 2001 & 2002 & 2003 & 2004 & 2005 & 2006 & 2007 & 2008 & 2009 & 2010 \\
\hline Argentina & $7,8 \%$ & $10,5 \%$ & $14,2 \%$ & $19,7 \%$ & $12,8 \%$ & $9,5 \%$ & $10,2 \%$ & $14,4 \%$ & $16,5 \%$ & $14,7 \%$ & $14,2 \%$ \\
\hline Bolivia & & & & & & & & & $29,8 \%$ & & $38,6 \%$ \\
\hline Brasil & $16,0 \%$ & $14,2 \%$ & $13,6 \%$ & $12,9 \%$ & $13,5 \%$ & $11,3 \%$ & $11,7 \%$ & $12,7 \%$ & $12,0 \%$ & $15,3 \%$ & $17,5 \%$ \\
\hline Chile & & & $15,7 \%$ & $16,9 \%$ & & $11,1 \%$ & $9,2 \%$ & $13,4 \%$ & $12,9 \%$ & $14,9 \%$ & $16,8 \%$ \\
\hline Colombia & & & & & & & $22,5 \%$ & $22,7 \%$ & $24,5 \%$ & $22,4 \%$ & $20,6 \%$ \\
\hline Costa Rica & & & & & & & & & & & $13,5 \%$ \\
\hline Ecuador & & & & & $27,2 \%$ & & & & $17,2 \%$ & $15,8 \%$ & $21,3 \%$ \\
\hline Guatemala & & & & & & & & & & $19,2 \%$ & $16,3 \%$ \\
\hline Jamaica & & & & & & $17,0 \%$ & $20,3 \%$ & & $15,6 \%$ & $22,7 \%$ & $10,5 \%$ \\
\hline
\end{tabular}

20\%, lo que hizo que en 2003 ya fueran 1.500, la mayoría de estas se caracteriza por ser sin ánimo de lucro (European Commission Enterprise Directorate-General, 2002).

${ }^{13}$ Otro ejemplo cercano es el Aburrá Norte, en el centro de Antioquia, Colombia, donde se muestra un crecimiento notable de la microempresa, entre 2000 y 2004, el 97\% de 5.657 empresas registradas son microempresas (el rango más bajo de activos, menos de un millón de pesos). 
Autoempleo y emprendimiento. Una hipótesis de trabajo para explicar una de las extrategias adoptadas por los gobiernos ...

\begin{tabular}{|l|l|l|l|l|l|l|l|l|l|l|l|}
\hline País & 2000 & 2001 & 2002 & 2003 & 2004 & 2005 & 2006 & 2007 & 2008 & 2009 & 2010 \\
\hline México & & $18,7 \%$ & $12,4 \%$ & & & $5,9 \%$ & $5,3 \%$ & & $13,1 \%$ & & \\
\hline Panamá & & & & & & & & & & $9,6 \%$ & \\
\hline Perú & & & & & $40,3 \%$ & & $40,1 \%$ & $25,9 \%$ & $25,6 \%$ & $20,9 \%$ & $27,2 \%$ \\
\hline Puerto Rico & & & & & & & & $3,0 \%$ & & & \\
\hline $\begin{array}{l}\text { República } \\
\text { Dominicana }\end{array}$ & & & & & & & & $16,8 \%$ & $20,4 \%$ & $17,5 \%$ & \\
\hline $\begin{array}{l}\text { Trinidad y } \\
\text { Tobago }\end{array}$ & & & & & & & & & & & $15,1 \%$ \\
\hline Uruguay & & & & & & & $12,6 \%$ & $12,2 \%$ & $11,9 \%$ & $12,1 \%$ & $11,7 \%$ \\
\hline Venezuela & & & & $27,3 \%$ & & $25,0 \%$ & & $20,2 \%$ & & $18,7 \%$ & \\
\hline
\end{tabular}

Fuente: Amorós (2011, p. 15).

\section{CONCLUSIONES Y RETOS PARA LA ACADEMIA Y EL GOBIERNO}

Como se vio a lo largo del trabajo, se asiste hoy a una nueva etapa del fomento del espíritu empresarial, donde se presenta un rápido crecimiento de las pequeñas empresas, en un contexto de reducción del Estado y ampliación del mercado, situación que contrasta con la vivida a principios del siglo XX donde de manera similar se tuvo un crecimiento de la cultura del autoempleo, pero donde, al contario de este tiempo, se vivía un crecimiento del Estado. La explicación a esta dinámica se debe, en uno y otro momento, entre otros factores, al interés del Gobierno por la eficiencia económica, y los factores sociales y políticos. El fomento de la cultura emprendedora ha resultado una buena estrategia de los gobiernos por mantener el orden social y la gobernabilidad, estrategia que es coherente con la nueva idea de gobernanza del desarrollo, donde se le da un papel preponderante al gobierno descentralizado para incidir positivamente el empleo, la competitividad y la equidad.

La literatura económica ha tratado de encontrar otras explicaciones al rebrote de la cultura emprendedora; se enfatiza en la idea de destrucción creativa, amor al riesgo, búsqueda de ganancia o como estrategia para conseguir empleo. En este texto se dejó planteada la hipótesis de que la difusión de esta cultura es fruto de la acción decidida del Gobierno, primero desde lo local -con apoyo de las universidades, agencias de desarrollo y del sector productivo- y luego desde lo nacional. Esta estrategia le ha resultado demasiado funcional y conveniente al Gobierno, pues el fomento de la cultura emprendedora ha producido un aumento de la productividad, a la vez que un aumento del bienestar social, y reducido el desempleo. 
De ser aceptada la invitación a trabajar sobre esta hipótesis, los campos de estudios que se abren para la academia son diversos; aquí se dejan planteados tres: el primero en el que se podría trabajar es sobre las políticas públicas sobre el autoempleo en el mundo, estudios que brinden la posibilidad de explorar las razones de fondo que expliquen por qué los Estados capitalistas modernos adoptaron una política pública de emprendimiento o autoempleo. Una buena forma de investigar sobre el tema consiste en hacer una evaluación objetiva y crítica de la política pública de los incentivos al emprendimiento empresarial en un país o región; por ejemplo, algunos países del sudeste asiático donde a finales de la década de 1980 se consolidó una fuerte política pública de emprendimiento, análisis que podría ser transversal con el examen de programas de lucha contra la pobreza que tienen entre sus estrategias el fomento de la las actividades de innovación y desarrollo empresarial.

El segundo campo de trabajo estaría en las ideas dejadas en la sección seis donde se insinuó que los gobiernos locales han tenido que asumir las responsabilidades del Gobierno nacional, como necesidad de incidir en el desarrollo económico de su territorio, a través de programas de fomento de la cultura del emprendimiento. Y el tercer campo de trabajo se relaciona con la política y la Administración Pública: se estudiaría la idea de cómo un programa público de estímulo al emprendimiento es una forma de mantener la gobernabilidad y la gobernanza.

Estos estudios necesitan un tratamiento riguroso, donde a través de cifras, estudios de caso, indicadores de gestión, de resultados, de impacto, de eficiencia o estimaciones econométricas, entre otras muchas estrategias metodológicas, se logre desarrollar tales ideas. Sin embargo, los retos no solo son para la academia; la Administración Pública también los tiene. El Gobierno ha logrado mostrar que mediante políticas de fomento del espíritu emprendedor y de cambios institucionales se puede dinamizar la actividad empresarial, aunque también es evidente la dificultad para que las empresas creadas se mantengan; de allí el reto que tiene el Gobierno hoy en este ámbito. El Gobierno debe hacer esfuerzos para consolidar la cultura emprendedora: cambios institucionales que influyan en la generación de iniciativas empresariales (Baumol, 1990; Bowen y De Clercq, 2008), conducentes a crear ambientes más propicios para la división del trabajo, la creación de ideas de negocios, la comercialización de la invención y la participación del sector privado. Pero también acciones que ayuden a los emprendedores a orientarse a actividades más productivas, que generen mayores puestos de trabajo y mayor valor.

Lo anterior se puede lograr a través de la protección de la libertad comercial, derechos de propiedad, los contratos aplicables, el fomento de oportunidades para 
Autoempleo y emprendimiento. Una hipótesis de trabajo para explicar una de las extrategias adoptadas por los gobiernos ...

las empresas de base, formación de capital humano y mejorar la disponibilidad de tecnología (ACS y Szerb, 2007; Arenius y Minniti, 2005). También, con la ampliación de recursos para fomentar nuevas empresas (capital de riesgo), aumento de créditos, mejorar la experiencia de los emprendedores en gerencia, luchar contra la débil participación del sector privado y contra la idea del mercado de que el emprendimiento es una conducta inapropiada (Cumming, 2007).

Para finalizar, de manera específica para Colombia, falta un mayor esfuerzo del Gobierno central por convertir el emprendimiento en una política de Estado. Aunque se han realizado esfuerzos recientes del Gobierno por fomentar el emprendimiento (Conpes No 3484 de 2007 y Ley 1014 de 2006), falta mayor trabajo desde los gobiernos regionales y locales, por llevar al emprendimiento informal al formal, del emprendimiento de subsistencia al de alto potencial de crecimiento y del emprendimiento aislado al emprendimiento en red, que permita generar economías de escala, desarrollar conocimiento aplicado y competir en el mundo.

\section{BIBLIOGRAFÍA}

Acs, Zoltan y Catherine Armington (1993). Conclusion. An: Acs, Zoltan J. and David B. Audretsch, eds. (1993). Small Firms and Entrepreneurship: An East-West Perspective. Cambridge, Mass, Cambridge University Press, 260p.

Acs, Zoltan y Catherine Armington (2004). Employment growth and entrepreneurial activity in cities. En: Regional Studies, Vol. 38, N. ${ }^{\circ}$ 8, pp. 911-927.

Acs, Zoltan y Laszlo Szerb (2007). Entrepreneurship. Economic Growth and Public Policy. En: Small Business Economics, Vol. 28, N. ${ }^{\circ} 2-3$, pp. 109-122.

Álvarez Herranz, Agustín; Pilar Valencia de Lara; Santiago Barraza y Ana María Legato (2010). Factors determining the entrepreneurial consolidation in Latin America. En: African Journal of Business Management, Vol. 4, N. . 9, pp. 1.717-1.722.

Amorós, José Ernesto (2011). El Proyecto Global Entrep reneurship Monitor (GEM): Una Aproximación Desde el Contexto Latinoamericano. En: Academia. Revista Latinoamericana de Administración, N. ${ }^{\circ}$ 46, pp. 1-15.

Amorós, José Ernesto y Oscar Cristi (2008). Entrepreneurship and competitiveness development: A longitudinal analysis of Latin American countries. En: International Entrepreneurship and Management Journal, Vol. 4, N. ${ }^{\circ}$ 4, pp. 381-399.

Amorós, José Ernesto; Fernández, Cristóbal y Tapia, Juan (2012). Quantifying the Relationship between Entrepreneurship and Competitiveness Development Stages in Latin America. En: International Entrepreneurship and Management Journal, Vol. 8, N. ${ }^{\circ}$, pp. 249-270.

Semestre Económico, volumen 15, №. 32, pp. 103-128 • ISSN 0120-6346, julio-diciembre de 2012, Medellín, Colombia 
Antelo, Eduardo (2006). Impulso a la creación de nuevos negocios. Proyecto de incubadora de empresas en Bolivia. Bogotá, D. C., Programa Andino de Cooperación (PAC), Corporación Andina de Fomento, Vol. 2, Serie: Colección PAC, 73p.

Arenius, Pia y Minniti, Maria (2005). Perceptual variables and nascent entrepreneurship. En: Small Business Economics Journal, Vol. 24, N.․ 3, pp. 233-247.

Audretsch, David; Martin Carree y Roy Thurik (2001). Does entrepreneurship reduce unemployment? Tinbergen Institute Discussion Paper TI 2001 -074/3, Rotterdam, Erasmus University Rotterdam, 12p.

Audretsch, David y Roy Thurik (2000). Capitalism and democracy in the 21st century: from the managed to the entrepreneurial economy. En: Journal of Evolutionary Economics, Vol. 10, N. ${ }^{\circ} 1, \mathrm{pp} .17-34$.

Audretsch, David y Roy Thurik (2001). What is new about the new economy: sources of growth in the managed and entrepreneurial economies. En: Industrial and Corporate Change, Vol. 19, pp. 795-821.

Aydalot, Philippe (1985). Économie Régionale et Urbaine, Economica, Paris, 487p.

Baumol, William (1990). Entrepreneurship: productive, unproductive and destructive. En: The Journal of Political Economy, Vol. 98, N.․ 5, Part. 1, pp. 893-921.

Baumol, William (1993). Entrepreneurship, management, and the structure of payoffs. Cambridge, MA, MIT-Press, XI, 311p.

Beck, Urich (1998). La sociedad del riesgo. Barcelona, Paídos, 304p.

Birch, David (1979). The job creation process. Program on Neighborhood and Regional Change. Cambridge, Mass, MIT Press, 302p.

Blau, David (1987). A time series analysis of self-employment. En: Journal of Political Economy, N. ${ }^{\circ} 95$, pp. $445-467$.

Brock, William y David Evans (1989). Small business economics. En: Small Business Economics, Vol. 1, N. ${ }^{\circ}$ 1, pp. 7-20.

Bowen, Harry y Dirk De Clercq (2008). Institutional context and the allocation of entrepreneurial effort. En: Journal of International Business Studies, Vol. 39, N. ․ 4, pp. 747-767.

Cantillon, Richard (1950 [1755]). Ensayo sobre la Naturaleza del Comercio en General. México, Fondo de Cultura Económica, 234p.

Capelleras, Joan-Lluis; Francis Greene; Hugo Kantis y Rodrigo Rabetino (2010). Venture creation speed and subsequent growth: Evidence from South America. En: Journal of Small Business Management, Vol. 48, N.․ 3, pp. 302-324.

Carlsson, Bo (1989). The evolution of manufacturing technology and its impact on industrial structure: an international study. En: Small Business Economics Vol. 1, ․․ 1, pp. $21-38$. 
Autoempleo y emprendimiento. Una hipótesis de trabajo para explicar una de las extrategias adoptadas por los gobiernos ...

Carlsson, Bo (1992). The rise of small business; causes and consequences. En: William James Adams (ed.). Singular Europe, Economy and Policy of the European Community after 1992. Ann Arbor: University of Michigan Press, pp. 145-169.

Chandler, Alfred (1977). The Visible Hand: The Managerial Revolution in American Business. Cambridge, Mass, Harvard University Press, 608p.

Conpes -Consejo Nacional de Política Económica y Social- (2007). Política nacional para la transformación productiva y la promoción de las micro, pequeñas y medianas empresas: un esfuerzo público-privado. Documento Compes 3484. Bogotá, D. C., Ministerio de Comercio, Industria y Turismo, Departamento Nacional de Planeación-Dirección de Desarrollo Empresarial, 13 de agosto de 2007, 32p.

CRC- Congreso de la República de Colombia- (2006). Ley 1014 de 2006 del 26 de enero de 2006, por la cual se da fomento a la cultura del emprendimiento.

Cressien, John (2006). Espíritu empresarial como estrategia de competitividad y desarrollo. En: Revista EAN, N. ${ }^{\circ}$ 7, pp. 103-118.

Cumming, Douglas (2007). Government policy towards entrepreneurial finance: Innovation investment funds. En: Journal of Business Venturing, Vol. 22, N.․ 2, pp. 193-235.

Delvalle, Manuel (2006). Forjando emprendedores. Las incubadoras de empresas en el Ecuador. Bogotá D. C., Programa Andino de Cooperación (PAC), Corporación Andina de Fomento, Vol. 3, Serie: Colección PAC, 74p.

European Commission Enterprise Directorate-General (2002). Benchmarking of Business Incubators. [En línea] http://ec.europa.eu/enterprise/index _ en.htm [octubre 20 de 2010].

Figarella, Dayana y Andres Zamora (2006). Semillero empresarial. El servicio de Atención al Emprendedor de Venezuela (SAE). Bogotá, D. C., Programa Andino de Cooperación (PAC), Corporación Andina de Fomento, Vol. 4, Serie: Colección PAC, 62p.

Foelster, Stefan (2000). Do entrepreneurs create jobs? En: Small Business Economics, N. ${ }^{\circ} 14$, pp. $137-148$.

Galbraith, John (1956). American Capitalism: the Concept of Countervailing Power. Boston, Houghton Mifflin Co., 208p.

García, Manuel (2002). Autoempleo y trabajo asociado: el trabajo en la economía social. Córdoba, Ediciones de la Universidad de Córdoba, 360p.

Godin, Keith; Jason Clemens y Niels Veldhuis (2008). Easuring entrepreneurship: Conceptual frameworks and empirical indicators En: Studies in Entrepreneurship and Markets, N. ${ }^{\circ} 7$. Fraser Institute, 66p.

Grossman, Gene y Elhanan Helpman (1991). Innovation and Growth in the Global Economy. Cambridge, Mass, MIT Press, xiiv+359p.

Semestre Económico, volumen 15, №. 32, pp. 103-128 • ISSN 0120-6346, julio-diciembre de 2012, Medellín, Colombia 
Guzman, Joaquín e Isidro Romero (2005). "La trascendencia económica del trabajo autónomo". En: Temas Laborales, N. ${ }^{\circ} 81$, pp. 79-97.

Johannisson, Bengt (1995). Paradigms and entrepreneurial networks-some methodological challenges. En: Entrepreneurship and Regional Development Vol.7, pp. 215-231.

Kantis, Hugo (2004). Main contrasts between Latin America and East Asia, Italy and Spain. En: Hugo Kantis, Pablo Angelelli and Virginia Moori-Koenig (Eds.). Experience in Latin America and worldwide 2004. Washington, D. C.: Inter-American Development Bank-Fundes International, pp. 57-74.

Kantis, Hugo (2005a). The emergence of dynamic ventures in Latin America, Southern Europe and East Asia: An international comparison. En: International Journal of Entrepreneurship and Small Business, Vol. 2, N. ${ }^{\circ}$ 1, pp. 34-56.

Kantis, Hugo, editor, con la colaboración de Pablo Angelelli y Virginia Moori Koenig (2005b). Desarrollo emprendedor, América Latina y la experiencia internacional. New York, Banco Interamericano de Desarrollo-BID-, 277p.

Kantis, Hugo; Masahiko Ishida y Masahiko Komori (2002). Entrepreneurship in emerging economies: The creation and development of new firms in Latin America and East Asia. Washington, D. C., Inter-American Development Bank, 132p.

Kirzner, Israel (1997). Entrepreneurial discovery and the competitive market process: an Austrian approach. En: Journal of Economic Literature, Vol. 35, pp. 60-85.

Knight, Frank (1999). Selected Essays by Frank H. Knight. Vol. 1: What Is Truth in Economics? Edited by Ross Emmett. Chicago, University of Chicago Press, 1999. xxiv; 406p.

Loveman, Gary y Wernerand Sengenberger (1991). "The re-emergence of small-scale production; an international comparison". En: Small Business Economics, Vol. 3, N. ${ }^{\circ}$, pp. 1-37.

Lucas, Robert (1988). On the mechanics of economic development. En: Journal of Monetary Economics, N. ${ }^{\circ} 22$, pp. 3-39.

Lucas, Robert (1993). Making a miracle. En: Econometrica, Vol. 61, N. ${ }^{\circ} 2$, pp. 251-272.

Martín, Hans-Peter y Harald Schumann (2000). La trampa de la globalización. Madrid, Taurus, 319p.

Marx, Karl (2001). El Capital, Vols. 1-8, 24a Ed., México, Siglo XXI.

Organización Internacional del Trabajo (OIT), Centro de Información de las Naciones Unidas (2009). El número de desempleados, trabajadores pobres y empleos vulnerables aumentará de manera espectacular debido a la crisis económica mundial. [En línea] 
Autoempleo y emprendimiento. Una hipótesis de trabajo para explicar una de las extrategias adoptadas por los gobiernos ...

Comunicado de prensa, CINU/005. 〈 http://unic.un.org/imucms/userfiles/asuncion/file/ cinu005.09.doc. $\rangle$ |enero 28 de 2009].

Peres, Wilson y Giovanni Stumpo, coords. (2002). Pequeñas y medianas empresas industriales en América Latina y el Caribe. México D.F., CEPAL/ Siglo XXI, 549p.

Piore, Michael y Charles Sabel (1984). The Second Industrial Divide: Possibilities for Prosperity. New York, Basic Books, 280p.

Rifkin, Jeremy (1995). The End of Work. New York, Tarcher/Putnam, 312p.

Rodríguez, Carlos y Manuel Jiménez, (2005). Emprenderismo, acción gubernamental y academia: revisión de la literatura. En: Innovar Vol.15, ․․ 26, pp. 73-89.

Romer, Paul (1986). Increasing returns and long-run growth. En: Journal of Political Economy, Vol. 94, N. ${ }^{\circ}$, pp. 1.002-1.037.

Saxenian, AnnaLee (1990). Regional networks and the resurgence of Silicon Valley. En: California Management Review, N. 33 , pp. 89-111.

Saxenian, AnnaLee (1994). Regional Advantage: Culture and Competition in Silicon Valley and Route 128. Cambridge, Mass, Harvard University Press, 240p.

Schumpeter, Joseph (1934). The Theory of Economic Development. Cambridge, Mass, Harvard University Press, lxiv, 255p.

Schumpeter, Joseph (1942). Capitalism, Socialism and Democracy. New York, Harper and Row, 598p.

Shane, Scott (2000). Prior knowledge and the discovery of entrepreneurial opportunities. En: Organization Science, N..$^{\circ} 11$, pp. 448-469.

Smith, Adam (1996 [1776]). La Riqueza de las naciones. Madrid, Alianza Editorial, 818p.

Tiffin, Scott (2004). Entrepreneurship in Latin America. Westport, CT: Praeger, 322p.

Valencia Agudelo, Germán Darío (2004). Metamorfosis del Estado: de empresario a regulador: El caso de los servicios públicos domiciliarios en Colombia. En: Ecos de Economía, N. 18, pp. 9-32.

Van Stel, André (2006). Empirical Analysis of Entrepreneurship and Economic Growth. New York, International Studies in Entrepreneurship, Vol. 13, Springer Science, 240p.

Varela, Rodrigo y Olga Lucía Bedoya (2006), Modelo conceptual de desarrollo empresarial basado en competencias. En: Estudios Gerenciales, Vol. 22, N. ${ }^{\circ}$ 100, pp. 21-47.

Vázquez, Antonio (2005). Las nuevas fuerzas del desarrollo. Barcelona, Antoni Bosch, 177p.

Wennekers, Sander y Roy Thurik (1999). Linking entrepreneurship and economic growth. En: Small Business Economics, Vol. 13, N. ${ }^{\circ}$ 1, pp. 27-55.

Semestre Económico, volumen 15, №. 32, pp. 103-128 • ISSN 0120-6346, julio-diciembre de 2012, Medellín, Colombia 